\title{
Multiple cutaneous swellings in an immunocompetent host - cryptococcosis overlooked
}

\section{Authors}

Kranthi Kosaraju ${ }^{1}$

Chiranjoy

Mukhopadhyay ${ }^{2}$

Vandana $\mathrm{KE}^{3}$

Kiran Yagain ${ }^{4}$

Rao $\mathrm{NR}^{5}$

${ }^{1}$ Assistant Professor,

Department of

Microbiology, Kasturba

Medical College, Manipal

University, Manipal, India

${ }^{2}$ Professor and Head,

Department of

Microbiology, Kasturba

Medical College, Manipal

University, Manipal, India

${ }^{3}$ Associate Professor,

Department of

Microbiology, Kasturba

Medical College, Manipal

University, Manipal, India

${ }^{4}$ Associate Professor,

Department of Pathology,

Kasturba Medical College,

Manipal University,

Manipal, India

${ }^{5}$ Professor, Department of

General Medicine, Kasturba

Medical College, Manipal

University, Manipal, India

Submitted on: $1 / 6 / 201$ Approved on: $2 / 6 / 2011$

Correspondence to: Kranthi Kosaraju Department of

Microbiology Kasturba Medical College,

Manipal, 575104

medkranthi@gmail.com

We declare no conflict of interest.

(2011 Elsevier Editora Ltda. All rights reserved.

\begin{abstract}
Recently, there has been an increase in the incidence of cryptococcosis even among immunocompetent patients. We present a case of multiple subcutaneous swellings later developing into a nonhealing ulcer caused by Cryptococcus in a 60 year-old immunocompetent male along with a brief review of clinical presentations of cutaneous cryptococcosis in immunocompetent and immunocompromised individuals. A high index of suspicion is desired in view of possible dissemination and variable non-specific clinical presentations.
\end{abstract}

Keywords: cryptococcosis; Cryptococcus neoformans; skin ulcer.

\section{INTRODUCTION}

Cutaneous cryptococcosis is rarely seen in immunocompetent hosts, with protean clinical manifestations making clinical diagnosis difficult. ${ }^{1,2}$ We report a rare case of cutaneous cryptococcosis in a immunocompetent patient without clinical or laboratory evidence of dissemination.

\section{CASE REPORT}

A 60-year male timber merchant presented with history of perianal wound on right side with non-foul smelling discharge for three months. Initially it had begun as a small swelling which later ulcerated. He also had a swelling over the sacral region almost at the same time as that of the swelling in perianal region, both of which grew over a period of a month. The swelling over sacral region was spontaneous in onset, slowly progressing in size over two weeks time, not preceded by trauma or injuries, for which he consulted a local surgeon. The sacral swelling was clinically diagnosed as lipoma and was excised while the perianal swelling was debrided and left open. Histopathology of the excised swelling revealed "fat necrosis with cryptococcal infection" (Figures 1 and 2). As the patient was discharged before the reports were available, specific antifungal therapy could not be started.

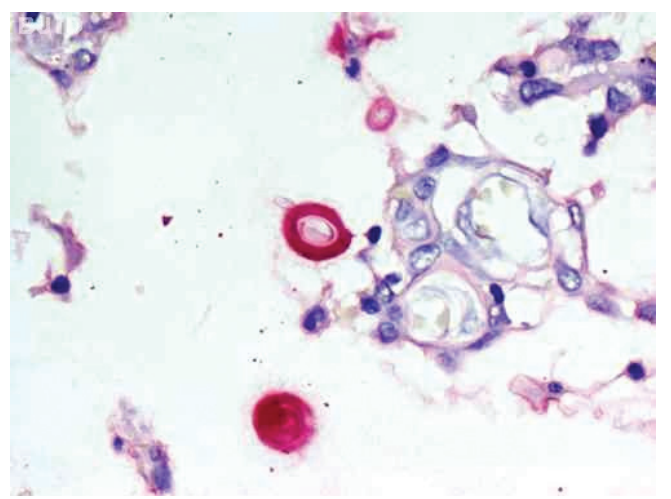

Figure 1: Mucicarmine stain highlighting the cryptococcal capsule, $400 \mathrm{x}$.

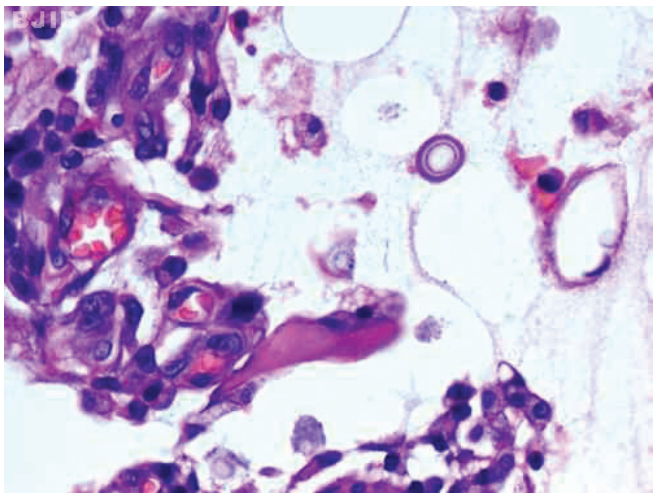

Figure 2: Hemotoxylin \& eosin stain, 400x. Cryptococcal organisms. 
During the current hospitalization, he had non-foul smelling discharge from both post-operative wound in sacral region which did not heal completely and from perianal wound, which also failed to heal even after three months. On examination, there was a vertical surgical wound over the sacral region, $10 \mathrm{~cm}$ horizontally, tender with swollen periphery. The perianal wound measuring $2 \times 3 \mathrm{~cm}$ was seen to the right of the anus. It was clean with little serous non-foul smelling discharge. He did not have diabetes, hypertension or any other illnesses in the past. He did not smoke or consumed alcohol. On examination he was afebrile, BP was $160 / 80 \mathrm{mmHg}$, abdomen was soft, no organomegaly noted, cardiovascular and respiratory systems were normal.

His routine investigations were $\mathrm{Hb}-14.3 \mathrm{~g} \%$, TLC $7000 / \mathrm{cmm}, \mathrm{N}-57.5 \%, \mathrm{~L}-30 \%, \mathrm{M}-10 \%, \mathrm{E}-7 \%, \mathrm{~B}-0 \%$, ESR - $7 \mathrm{~mm}$. Random blood glucose, urine routine, liver function tests were normal. Immunoglobulin levels and CD4 counts were within normal limits and ELISA for antibodies to HIV was negative.

Discharge from perianal wound was sent for routine microbiological culture. After $48 \mathrm{~h}$ of incubation at $37^{\circ} \mathrm{C}$ mucoid colonies were noted on Chocolate agar which on Gram stain showed Gram-positive spherical budding yeast cells with capsule. India ink from the culture confirmed capsule formation. The yeast was identified as Cryptococcus neoformans by standard microbiological procedures.

Based on the present culture results and previous histopathological report of the excised swelling, a diagnosis of cutaneous cryptococcosis was made. At this stage, he was interrogated about presence of any similar swellings and evidence of immunosuppression and dissemination of cryptococcosis. He recollected having a small swelling in the scalp above the right ear. On examination, a soft non tender fluctuant swelling of $1 \times 1 \mathrm{~cm}$ was noted. Interestingly, he also recollected to have had intense headache preceding the appearance of these multiple swellings. He also reported to have lost $5 \mathrm{~kg}$ of weight in three months. He denied taking steroids or any other medications.

The patient was investigated further for evidence of dissemination by performing blood cultures, serum antigen detection and chest radiographs, all of which did not provide any evidence of dissemination. As blood cultures and serum antigen were negative, obtaining CSF for culture and antigen detection was thought to be unnecessary at this stage.

Considering the clinical presentations of multiple skin lesions and history of headache earlier, the therapy was instituted as a case of disseminated cryptococcosis to prevent relapse(s). He was given amphotericin (0.7-1.0 $\mathrm{mg} / \mathrm{kg} /$ day) for 10 days but was soon replaced by fluconazole $400 \mathrm{mg} /$ day in view of deteriorating renal functions. At the time of writing this report, he had completed six weeks of specific therapy and was being followed up. The wound showed signs of healing, discharge had stopped and also there was regression in the size of swelling in scalp. The patient was advised to continue fluconazole $400 \mathrm{mg} /$ day for a total of eight weeks along with advice on follow-up of his immunological and HIV status.

\section{DISCUSSION}

Immunocompromised individuals constitute the majority of patients diagnosed with cryptococcosis; however, with improving antiretroviral agents for patients with AIDS, organ transplant recipients are at highest risk of acquiring this infection. ${ }^{3}$ Primary site of C. neoformans infection is usually the respiratory tract or the central nervous system, however, it may also involve cutaneous, ocular, or genitourinary systems. ${ }^{4}$ Skin is the most common extra-neural site of infection, affecting $10 \%-20 \%$ of those with systemic involvement. ${ }^{5}$

Skin involvement by Cryptococcus occurs either as a part of disseminated infection or solely as cutaneous form. Secondary cutaneous cryptococcosis results from haematogenous spread of the fungus while the primary cutaneous form occurs by transcutaneous introduction of the fungus. ${ }^{6}$ The disseminated variety, especially in patients with AIDS, is characterized by multiple lesions simulating molluscum contagiosum. ${ }^{7}$ Lesions usually occur in covered parts of the body with multicentric skin involvement. ${ }^{8}$ Cutaneous lesions are considered an ominous sign as they are often the first presenting symptom of systemic disease. ${ }^{9}$

However, PCC can occur in both immunocompetent and compromised patients. ${ }^{7}$ It usually presents as a solitary lesion on uncovered parts accompanied by regional lymph node involvement. ${ }^{8}$ Infection most commonly involves extremities, especially the upper-body extremities. Finger and facial sites were particularly common sites in non-immunocompromised hosts whereas immunocompromised hosts have multiple sites of infection or infection localized to trunk or lower extremities. ${ }^{7}$

A clinical diagnosis of cutaneous cryptococcosis is difficult to make due to lack of pathognomonic skin lesion. Cryptococcosis may present itself as erythematous swellings, nodular, cystic-appearing excrescences, granulomas, acneiform papules or pustules, crusted or infiltrating plaques, ulcers, or cellulitis. ${ }^{3}$ Cellulitis is more often a sign of dissemination than of primary cutaneous disease and if present in compromised patients, carries a high mortality of $28 \%-45 \%$.

Most commonly described lesions are nodule, ulcer and whitlow, ${ }^{1}$ however, subcutaneous soft swellings which later ulcerated was the presentation in our case.

Secondary work-up of patients with isolated cryptococcal skin lesions should be undertaken in all cases and should include evaluation for evidence of dissemination as well as for underlying causes of immunodeficiency. This includes serum cryptococcal antigen detection, blood, urine and spu- 
tum cultures, chest $\mathrm{x}$-ray, thorough dermatologic examination to look for similar lesions elsewhere, screening for HIV antibodies, CD4 counts, immunoglobulins estimation and blood glucose levels. For immunocompetent patients, if any of the initial work-up indicates disseminated disease or the symptoms are suggestive of CNS involvement, CSF should be tested for cryptococcal antigen and fungal culture should be performed. ${ }^{7}$ Lumbar puncture was not done in our case as serum antigen detection was negative at this stage when patient presented to us. Thorough work up in our case could not provide any evidence of primary site of infection or of dissemination. There was no history of trauma preceding the onset of these lesions and no evidence of immunosuppression. Negative blood cultures and serum antigen testing could be partly explained by immunocompetency of the host.

We could not prove dissemination in our patient but with the available evidence from literature, involvement of multiple cutaneous sites, as is in our case, often suggest disease dissemination instead of multiple sites of cutaneous inoculation. ${ }^{8}$ Since our patient was a timber merchant by occupation, any possible exposure to wood and dust could be a possible source of infection. An interesting feature was appearance of intense headache prior to onset, which if left untreated, can progress to overt meningitis and/or dissemination, and hence the need of treatment protocol as for disseminated disease. Appearance of headache before appearance of cutaneous lesions was also seen in similar case reports from the literature. ${ }^{2}$

Long term therapy and follow-up is recommended to prevent relapses since mortality of disseminated cryptococcosis is $70 \%-80 \%$ higher in untreated patients compared with those treated with systemic antifungal agents. ${ }^{5}$

\section{CONCLUSION}

Cutaneous cryptococcosis can present in a disseminated form without any evidence of immunosuppression. However, a thorough work up of all cases with skin involvement is suggested along with a long term follow up for patient's immunological status. Clinical presentation can often be misleading with varied presentations; however, histopathological and microbiological investigations will help to clinch the diagnosis.

\section{REFERENCES}

1. Yuge S, Bastazini Jr I, Coelho MCM, Soares CT. Cutaneous cryptococcosis in an immunocompetent host. Acta Dermatovenereologica 2006; 86(2):165-6.

2. Vijaya D, Anand Kumar BH, Nagarathnamma T. Case report. Disseminated cutaneous cryptococcosis in an immunocompetent host. Mycoses 2001; 44:113-4.

3. Moe K, Lotsikas-Baggili AJ, Kupiec-Banasikowska A, Kauffman CL. The cutaneous predilection of disseminated cryptococcal infection in organ transplant recipients. Arch Dermatol 2005; 141:913-4.

4. Mehrabi M, Bagheri S, Leonard MK, Perciaccante VJ. Mucocutaneous manifestations of cryptococcal infection: report of a case and review of literature. J Oral Maxillofac Surg 2005; 63:1543-9.

5. Dharmshale SN, Patil SA, Gohil A et al. Disseminated cryptococcosis with extensive cutaneous involvement in AIDS. Indian J Med Microbiol 2006; 24(3):228-30.

6. Severo LC, Zardo IB, Londero AT. Cutaneous cryptococcosis due to Cryptococcus neoformans var. gattii. Rev Iberoam Micol 2001; 18:200-1.

7. Christianson JC, Engber W, Andes D. Primary cutaneous cryptococcosis in immunocompetent and immunocompromised hosts. Med Mycol 2003;41:177-88.

8. Tilak R, Prakash P, Nigam C et al. Cryptococcal meningitis with an antecedent cutaneous cryptococcal lesion. Dermatol Online J 2009; 15 (9):12.

9. Quartarolo N, Thomas I, Li H et al. Cutaneous cryptococcosis. Acta Dermatovenerologica 2002;11(4). 